\title{
Análise do número de cerdas internas do órgão de Haller de larvas de Amblyomma nodosum Neumann (Acari, Ixodidae)
}

\author{
Karla Bitencourth ${ }^{1}$, Rodrigo Hidalgo Friciello Teixeira ${ }^{2}$, Marinete Amorim ${ }^{1}$, \\ Gilberto Salles Gazêta ${ }^{1} \&$ Nicolau Maués Serra-Freire ${ }^{1}$
}

${ }^{1}$ Laboratório de Ixodides, Departamento de Entomologia, Instituto Oswaldo Cruz/Fiocruz, 21045-900 Manguinhos, Rio de Janeiro-RJ, Brasil. mamorim@ioc.fiocruz.br

${ }^{2}$ Parque Zoológico Municipal Quinzinho de Barros, 18021-020 Rua Theodoro Kaizel-Sorocaba, São Paulo-SP, Brasil.

\begin{abstract}
Analysis of the number of internal setae in Haller's organ of larvae of Amblyomma nodosum Neumann (Acari, Ixodidae). Larvae from Amblyomma nodosum Neumann, 1899 from engorged female ticks removed from Tamandua tetradactyla (Linnaeus, 1758) were obtained under laboratory conditions. Two hundred and nineteen larvae were examined under brightfiel microscopy with and imersion objective and confocal laser scanning microscopy in order to analyze the variation of the number of internal setae of the right and left side of the Haller's organ chamber. The mean number of internal setae was 5,06 $\pm 0,71$, with parametric valves of 4-11 setae.
\end{abstract}

KEYWORDS. Wild hosts; immature stage; ixodids; mammals; tick.

RESUMO. Análise do número de cerdas internas do órgão de Haller de larvas de Amblyomma nodosum Neumann (Acari, Ixodidae). A partir de teleógina de Amblyomma nodosum Neumann, 1899, removida de Tamandua tetradactyla (Linnaeus, 1758) foram obtidas posturas que originaram larvas, em condições de laboratório. Um total de 219 larvas foi examinado em microscopia de luz, com objetiva de imersão e microscopia de confocal com varredura a laser. Buscou-se analisar a variação do número das cerdas internas dos lados direito e esquerdo, no fundo da cápsula do Órgão de Haller, com intuito de utilizá-las como um parâmetro quetotáxico na diagnose específica. A média do número de cerdas foi 5,06 $\pm 0,71$, com valores limites da amplitude de 4-11.

PALAVRAS-CHAVE. Carrapato; estádio imaturo; ixodídeos; hospedeiros silvestres; mamífero.

Amblyomma nodosum foi proposta por Neumann (1899) com base na morfologia dos estádios adultos e ninfas. A ocorrência desses carrapatos foi registrada pela primeira vez na Costa Rica e, posteriormente, na Guatemala, Panamá, México, Nicarágua, Bolívia, Trinidad, Venezuela, Colômbia e Brasil (Robinson 1926; Vogelsang \& Santos Dias 1953; Keirans \& Brewster 1981; Serra-Freire et al. 1993, Manzanilla et al. 2002).

No Brasil, A. nodosum foi assinalada nos Estados do Rio de Janeiro, Minas Gerais, São Paulo, Goiás, Mato Grosso do Sul, Mato Grosso, Paraná e Rio Grande do Sul (Rohr 1909; Aragão 1936; Evans et al. 2000, Martins et al. 2004; Arzua et al. 2005). Todas as vezes que houve registros dessa espécie, foi assinalada parasitando tamanduás, seja tamanduá-mirim (Tamandua tetradactyla Linnaeus, 1758), ou tamanduábandeira (Myrmecophaga tridactyla Linnaeus, 1758) (Rohr 1909; Serra-Freire et al. 1993).

São poucos os estudos referentes às larvas do gênero Amblyomma, especialmente no Brasil. Geralmente, os trabalhos estão relacionados à descrição morfológica (Amorim \& SerraFreire 2000; Famadas et al. 1997; Amorim et al. 1997, BarrosBattesti et al. 2005), à presença de cerdas no interior da cápsula halleral e à sua morfologia (Amorim et al. 2001).

O órgão de Haller é um dos mais extraordinários sistemas sensoriais nos Ixodidae exibindo considerável diversidade, seja em nível de gênero ou espécie (Wooley 1988) e por isso suas características externas e internas têm sido utilizadas como carateres diagnósticos (Balashov \& Leonovich 1978; Homsher \& Sonenshine 1979; Hess \& Vilmant 1983 a, b; Estrada- Peña et al. 1986, Amorim et al. 2001, 2002; Sant'Anna et al. 2003). Este órgão exibe forma de fenda quando fechado e quase esférico, globoso, quando aberto, estando situado na superfície dorsal do tarso do primeiro par de patas dos carrapatos. O órgão é constituído por uma depressão em forma de cápsula com uma membrana fina e transparente, e numerosas estruturas cuticulares pleomorfas alongadas em seu interior (Balashov $\&$ Leonovich 1978), que exerce importante papel na busca e na detecção pelo sítio de fixação no hospedeiro. Estrada-Peña $e t$ al. (1986) concluíram que a provável função das cerdas internas da cápsula do órgão de Haller está relacionada com a captação de estímulos hormonais, já que a sua posição no interior do órgão proporciona ao carrapato grande proteção em relação a fatores externos. Axtell (1979) reportou que a capacidade quimiorreceptora dessas cerdas, provavelmente estaria ligada à capacidade olfativa dos carrapatos, confirmando a informação de Balashov (1972), posteriormente respaldada por Wooley (1988) e Sonenshine (1991).

O presente estudo tem como objetivo analisar o número de cerdas internas dos lados direito e esquerdo da cavidade posterior do Órgão de Haller de larvas de A. nodosum, com a perspectiva de utilizá-las como um parâmetro quetotáxico de diagnose específica. 


\section{MATERIALE MÉTODOS}

Teleóginas de A. nodosum, removidas de Tamandua tetradactyla (tamanduá-mirim), foram identificadas, através de chave dicotômica de Aragão \& Fonseca (1961) e Robinson (1926). A partir destas fêmeas, foram obtidas posturas que originaram neolarvas, em condições de laboratório. Um dia após a eclosão, as neolarvas foram sacrificadas em água quente $\left( \pm 70^{\circ} \mathrm{C}\right)$, preservadas em etanol $70^{\circ} \mathrm{GL}$ e trabalhadas para montagem definitiva, entre lâmina e lamínula, segundo técnica descrita por Amorim \& Serra-Freire (1995). Foram analisadas 219 larvas, com exame das cerdas internas da cavidade posterior do Órgão de Haller dos lados direito e esquerdo, ao microscópico de luz com objetiva de imersão no Laboratório de Ixodides, Departamento de Entomologia/Instituto Oswaldo Cruz (IOC)/Fundação Oswaldo Cruz (FIOCRUZ) e por microscopia de confocal com varredura a laser, no Departamento de Patologia-IOC, a fim de confirmar as contagens realizadas em microscopia de luz.

Para a análise estatística do número de cerdas internas foram utilizadas as medidas de tendência central: Média $\left(\mathbf{x}^{\mathbf{}}\right)$, Mediana (md) e Moda (mo), e de dispersão: Desvio Padrão (s), número menor (a) e número maior (A), para comparação de médias entre os lados. Também foram calculados os coeficientes de assimetria (AS) e de curtose (CC) (Serra-Freire 2002).

\section{RESULTADOS}

Em 219 larvas de A. nodosum (Fig. 1) foram observadas 2224 cerdas no interior do Órgão de Haller (Fig. 2), sendo 1110 do lado direito e 1114 do lado esquerdo. Houve variação do número de cerdas entre os lados analisados de um mesmo espécime (Tabela I). No lado direito, o menor número de cerdas encontrado (a) foi de quatro (4) e o maior (A) de dez (10) com número médio ( $\left.\mathbf{x}^{-}\right)$de 5,06 cerdas e desvio padrão (s) de 0,71 (Tabela II). No lado esquerdo $\mathbf{a}=4, \mathbf{A}=11, \mathbf{x}^{-}=5,08$ e $\mathbf{s}=0,69$. Os dois lados apresentaram moda (mo) e mediana (md) igual a
Tabela I. Freqüência absoluta e relativa de larvas de Amblyomma nodosum Neumann, 1899, em relação ao número de cerdas internas do Órgão de Haller dos lados direito e esquerdo.

\begin{tabular}{rrrrrr}
\hline & \multicolumn{2}{c}{ Larvas } & \multicolumn{3}{c}{ Número de cerdas } \\
& $\mathrm{N}^{\circ}$ & $(\%)$ & LD & LE & Total \\
\hline 01 & $(0,46)$ & 5 & 8 & 13 \\
01 & $(0,46)$ & 8 & 7 & 15 \\
& 01 & $(0,46)$ & 10 & 8 & 18 \\
01 & $(0,46)$ & 9 & 11 & 20 \\
& 02 & $(0,91)$ & 6 & 6 & 12 \\
& 02 & $(0,91)$ & 8 & 6 & 14 \\
& 03 & $(1,37)$ & 8 & 8 & 16 \\
& 05 & $(2,28)$ & 5 & 6 & 11 \\
& 06 & $(2,74)$ & 4 & 4 & 8 \\
& 15 & $(6,85)$ & 5 & 4 & 9 \\
& 182 & $(83,10)$ & 5 & 5 & 10 \\
\hline Total & 219 & $(100)$ & & & \\
\hline
\end{tabular}

$\mathbf{N}^{0}=$ Freqüência Absoluta, \% = Freqüência Relativa, LD = Lado Direito, $\mathbf{L E}=$ Lado Esquerdo

cinco cerdas (Fig. 3). O coeficiente de curtose (CC) foi igual a zero nos dois lados, que identifica as distribuições normais leptocúrticas, com pequena assimetria positiva, tendo o lado esquerdo coeficiente de assimetria (As) igual a 0,12 e o lado direito igual a 0,96 (Tabela II).

Houve assimetria entre o número de cerdas dos lados direito e esquerdo, em $26(11,87 \%)$ espécimes analisados, dentre os quais, 21 (80,77\%) larvas de A. nodosum apresentaram diferenças de apenas uma cerda entre os lados; quatro $(15,38 \%)$ de duas cerdas e apenas uma larva $(3,85 \%)$ apresentou diferença de três cerdas entre os lados (Tabela III). Dos 193 (88,13\%) espécimes simétricos, 182 (94,30\%) possuíam semelhança de cinco cerdas entre os dois lados analisados, seis $(3,11 \%)$ espécimes apresentaram simetria de quatro cerdas, três $(1,55 \%)$ com simetria de oito cerdas e somente duas $(1,04 \%)$ espécimes apresentaram semelhança de seis cerdas nos lados direito e esquerdo analisados (Tabela IV).
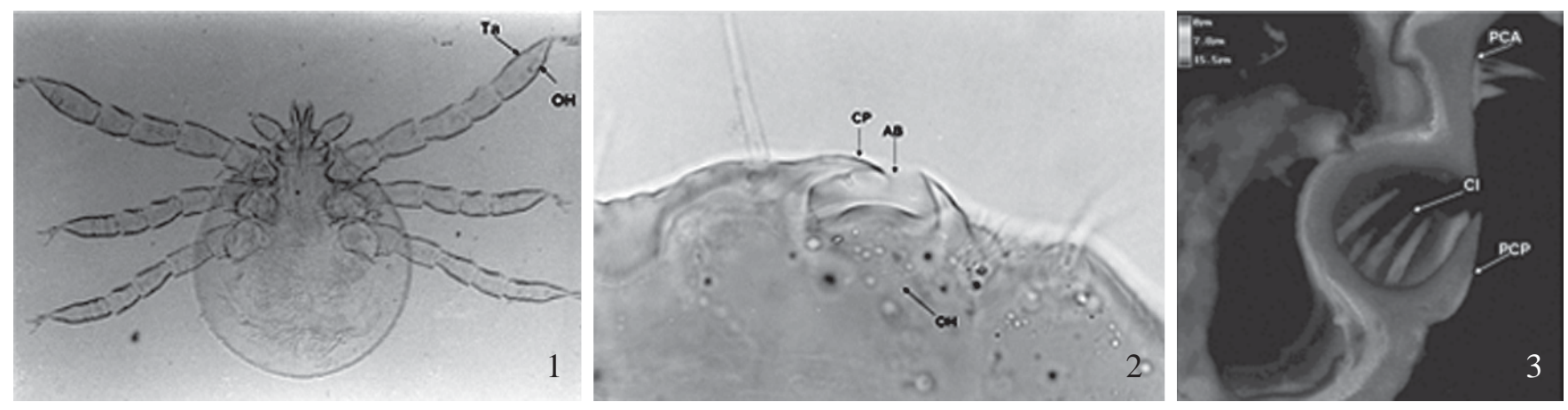

Figs. 1-3.1-2, Fotomicrografia da larva de Amblyomma nodosum Neumann, 1899. 1. Tarso I em vista dorsal, indicando o órgão de Haller. 2. Detalhe da cavidade posterior e da abertura do órgão de Haller em vista dorsal. 3. Confocal com varredura a laser, detalhe das cerdas internas, da parede da cavidade anterior e posterior do órgão de Haller. (Ta- Tarso I, OH- órgão de Haller, AB- Abertura da Cápsula Halleral, CP- Cavidade Posterior, PCA- Parede da Cavidade Anterior; PCP- Parede da Cavidade Posterior, CI- Cerdas Internas do Órgão de Haller). 
Tabela II. Medidas de tendência central e dispersão e coeficiente relativas ao número de cerdas internas do Órgão de Haller dos lados direito e esquerdo, de larvas de Amblyomma nodosum Neumann, 1899.

\begin{tabular}{lcc}
\hline & Lado Direito & Lado Esquerdo \\
\hline $\mathrm{a}$ & 4 & 4 \\
$\mathrm{~A}$ & 10 & 11 \\
$\overline{\mathrm{x}}$ & 5,06 & 5,08 \\
$\mathrm{md}$ & 5 & 5 \\
$\mathrm{mo}$ & 5 & 5 \\
$\mathrm{~s}$ & 0,71 & 0,69 \\
$\mathrm{As}$ & 0,12 & 0,96 \\
$\mathrm{CC}$ & 0 & 0 \\
\hline$\sum \mathrm{x}$ & 1110 & 1114 \\
\hline
\end{tabular}

$\mathbf{a}=$ Número menor, $\mathbf{A}=$ Número maior, $\overline{\mathbf{x}}=$ Média, $\mathbf{m d}=$ Mediana, $\mathbf{m o}$ $=$ Moda, $\mathbf{s}=$ Desvio Padrão, As $=$ Assimetria, $\mathbf{C C}=$ Coeficiente de Curtose $\sum \mathbf{x}=$ Somatório.

\section{DISCUSSÃO}

Roshdy et al. (1972) relataram que o Órgão de Haller dos Ixodidae apresenta sete cerdas típicas na cavidade anterior. Altern \& Prillinger (1980), reportaram de seis a sete cerdas sensorias em espécies da subfamília Amblyomminae e Ixodinae, mas Estrada-Peña et al. (1986), ressaltaram que existem somente de três a quatro cerdas no interior da cavidade posterior em larvas de Ixodidae. Estas diferenças entre espécies permitem sugerir que esse parâmetro quetotáxico possa subsidiar outros na diagnose específica de larvas de carrapatos.

Os resultados obtidos para larva de A. nodosum, referentes ao número de cerdas internas no Órgão de Haller, diferem dos de larvas de outras espécies da família Ixodidae e da família Argasidae, disponíveis na literatura (Homsher \& Sonenshine 1979; Altern \& Prillinger 1980; Estrada-Peña et al. 1986, Klompen \& Oliver 1993). Contudo, se assemelham ao reportado por Amorim et al. (2002) para Amblyomma geayi Neumann, 1899, diferindo na posição e na forma das cerdas. Desta forma, a amostra das larvas examinadas apresenta média, mediana e moda de cinco cerdas no interior desse órgão, nos lados analisados, em distribuição normal leptocúrtica, com pouca assimetria positiva. $\mathrm{O}$ resultado tem validade externa podendo ser referido para espécie como população e permite concordar com o relato de Amorim et al. (2001) que registraram

Tabela III. Freqüência absoluta e relativa de larvas assimétricas de Amblyomma nodosum Neumann, 1899, quanto ao número de cerdas internas do Órgão de Haller, dos lados direito e esquerdo.

\begin{tabular}{|c|c|c|c|}
\hline & \multicolumn{2}{|c|}{ Larvas } & \multirow{2}{*}{$\begin{array}{l}\text { Variação do número de cerdas entre } \\
\text { lados (assimetria) }\end{array}$} \\
\hline & $\mathrm{N}^{\mathrm{o}}$ & $(\%)$ & \\
\hline & 01 & $(3,85)$ & 03 \\
\hline & 04 & $(15,38)$ & 02 \\
\hline & 21 & $(80,77)$ & 01 \\
\hline Total & 26 & $(11,87)$ & \\
\hline
\end{tabular}

Tabela IV. Freqüência absoluta e relativa de larvas simétricas de Amblyomma nodosum Neumann, 1899, quanto ao número de cerdas internas do Órgão de Haller, dos lados direito e esquerdo.

\begin{tabular}{rrrrr}
\hline & \multicolumn{2}{c}{ Larvas } & LD & LE \\
\hline & $\mathrm{N}^{\text {o }}$ & $(\%)$ & & \\
& 2 & $(1,04)$ & 06 & 06 \\
& 3 & $(1,55)$ & 08 & 08 \\
& 6 & $(3,11)$ & 04 & 04 \\
& 182 & $(94,30)$ & 05 & 05 \\
\hline Total & 193 & $(88,13)$ & & \\
\hline
\end{tabular}

$\mathbf{N}^{0}=$ Freqüência Absoluta, $\%=$ Freqüência Relativa, $\mathbf{L D}=$ Lado Direito, $\mathbf{L E}=$ Lado Esquerdo

para o gênero Amblyomma a presença de duas a nove cerdas internas na cavidade posterior do órgão de Haller. Tal assertiva tem identidade com os resultados de Homsher \& Sonenshine (1979) para Amblyomma americanum (Linnaeus, 1758), com sete cerdas, e para Amblyomma variegatum (Fabricius, 1794), com seis. Andrade et al. (2005) encontraram quatro cerdas para Amblyomma cajennense (Fabricius, 1787).

As 11 cerdas como maior número em larva de $A$. nodosum difere do encontrado para $A$. geayi que apresentou sete cerdas (Amorim et al. 2002), de Anocentor nitens (Neumann, 1897) com oito (Sant'Anna et al. 2003) e de A. cajennense com cinco cerdas, sendo que essa variação interespecífica pode auxiliar na diagnose de larvas de ixodídeos.

\section{REFERÊNCIAS}

Altner, H. \& L. Prillinger. 1980. Ultrastructure of invertebrate chemotermo- and hygroreceptores and its significance. International Review Of Cytology 65: 69-139.

Amorim, M.; G. S. Gazêta \& N. M. Serra-Freire. 2002. Análise do número de cerdas internas do Órgão de Haller de larvas de Amblyomma geayi Neumann, 1899 (Acari: Ixodidae). Entomología y Vectores 9: 339-347.

Amorim, M.; G. S. Gazêta \& N. M. Serra-Freire. 2001. Estudo do Órgão de Haller de larvas de carrapato do gênero Amblyomma (Acari: Ixodidae) do Brasil. Entomología y Vectores. 8: 121-132.

Amorim, M. \& N. M. Serra-Freire. 2000. Morphological descripition of tick larval stage (Acari: Ixodidae). 7 Amblyomma auricularium (Conil. 1878). Entomología y Vectores 7: 297-310.

Amorim, M. \& N. M. Serra-Freire. 1995. Descrição morfológica do estádio de larva de carrapato (Acari: Ixodidae) 1. Amblyomma rotundatum Koch, 1844. Parasitologia Al Dia. 19: 09-19.

Amorim, M.; G. S. Gazêta; L. Guerim \& N. M. Serra-Freire. 1997. Morphological description of tick larval stage (Acari: Ixodidae). 5. Anocentor nitens (Neumann, 1897). Revista Brasileira de Parasitologia Veterinária 6: 143-156.

Andrade, C. C.; M. Amorim; G. S. Gazêta \& N. M. Serra-Freire. 2005. Número de cerdas internas do órgão de Haller em larvas de Amblyomma cajennense (Fabricius, 1787) (Acari: Ixodidae) nos municípios de Silva Jardim e Itaguaí, RJ. Arquivo Brasileiro Medicina Veterinária e Zootecnia, 57: 261-263.

Aragão, H. B. 1936. Ixodidas brasileiros e de alguns países limitrophes. Memórias do Instituto Oswaldo Cruz 31: 759-843.

Aragão, H. B. \& F. Fonseca. 1961. Notas de Ixodologia. VIII. Lista e chave para os representantes da fauna ixodológica brasileira. Memórias do Instituto Oswaldo Cruz 59: 115-29.

Arzua, M.; V. C. Onofrio \& D. M. Barros-Battesti. 2005. Catalogue of 
the tick collection (Acari, Ixodida) of the Museu de História Natural Capão da Imbuia, Curitiba, Paraná, Brazil. Revista Brasileira Zoologia 22: 623-632.

Axtell, R. C. 1979. Tarsal sensory receptors in ticks. In: Proceedings $4^{\text {th }}$ International Congress of Acarology (Saalfelden, 1974). Akadémiai Kiadó, Budapest p. 669-672.

Balashov, Yu. S. 1972. Bloodsucking ticks (Ixodoidea) -Vector of diseases of man and animals. Miscellaneous Publications of the Entomological Society of America 8: 1-376.

Balashov, Yu. S. \& S. A. Leonovich. 1978. Morphological characteristic of Haller's organ in tick of the tribe Amblyommatini (Acarina: Ixodidae). Entomological Review 55: 149-154.

Barros-Battesti, D. M.; M. Arzua; V. M. M. Rebello; F. S. Barbieri \& K. M. Famadas. 2005. Description of the larva of Amblyomma longirostre (Koch, 1844) (Acari: Ixodidae) by and electron microscopy. Revista Brasileira Parasitologia Veterinaria 14: $51-57$.

Estrada-Peña. J.; J. F. Gutierrez-Galindo; O. Sanchez-Acedo; B. OcaboMelendez; J. A. Castillo-Hernandez \& M. Galmes-Femenias. 1986. Morfologia del órgano de Haller en garrapatas (Ixodoidea). Revista Ibérica de Parasitología 46: 175-179.

Evans, D. E.; J. R. Martins; A. A. Guglielmone. 2000. A Review of the ticks (Acari: Ixodida) of Brazil, their hosts and geographic distribution- 1. The state of Rio Grande do Sul, southern Brazil. Memórias do Instituto Oswaldo Cruz 95: 453-470.

Famadas, K. M.; N. M. Serra-Freire \& R. M. Lanfredi. 1997. Redescription of the larvae of Amblyomma cajennense (Fabricius) (Acari:Ixodidae) using optical and scanning electron microscopy. Acarologia XXXVIII: 101-109.

Hess, E. \& M. Vlimant. 1983 a. The tarsal sensory system of Amblyomma variegatum Fabricius ( Ixodidae, Metastriata) II. No pore sensilla. Revue Suisse Zoologie 90: 157-167.

Hess, E. \& M. Vlimant. 1983 b. The tarsal system of Amblyomma variegatum Fabricius (Ixodidae, Metastriata). III Mapping of sensory hairs and evolution of the relative importance of sensory modalities during post-embryonic development. Revue Suisse Zoologie 90: 887-897.

Homsher, P. J. J. E. \& D. E. Sonenshine. 1979. Scanning electron microscopy of ticks for systematic studies: 3. Stucture of Haller's organ in five species of the subgenus Multidentatus of the genius Ixodes. In: Recent Advances in Acarology, II, J. G. Rodrigues (ed.). Academic Press, New York, p. 485-490.

Keirans, J. E. \& B. E. Brewster. 1981. The Nuttal and British Museum (Natural History) ticks collections lectotype designations for ticks (Acarina: Ixodoidea) described by Nuttal, Warburton, Cooper and
Robison. Bulletin of the British du Museum Naturelle d'Histoire, 41: 153-178.

Klompen, J. S. H. \& J. H. Oliver Jr. 1993. Haller's organ in the tick family Argasidae (Acari: Parasitiformes: Ixodida). Journal Parasitology, 79: 591-603.

Manzanilla, J.; M. E. García; R. E. Moissant; F. A. García \& E. Tortolero. 2002. Dos especies de garrapatas del género Amblyomma (Acari: Ixodidae) em perros del estado Aragua, Venezuela. EntomotropicaBoletín de Entomologia Venezolana, 17: 177-180.

Martins, J. R., I. M. Medri; C. M. Oliveira \& A.Guglielmone. 2004 Ocorrência de carrapatos em tamanduá-bandeira (Myrmecophaga tridactyla) e tamanduá-mirim (Tamandua tetradactyla) na região do Pantanal Sul Mato-Grossense, Brasil. Ciências Rural 34: 293295.

Neumann, L. G. 1899. Révision de la famille des ixodidés. Mémoire de la Société Zoologique de France 12: 107-294.

Robinson, L. E. 1926. Ticks a monograph of the Ixodoidea. IV. The genus Amblyomma. Cambridge Univ. Press., New York. 302 pp.

Rohr, C. J. 1909. Estudos sobre Ixodidas do Brasil. Ed. Gomes, Irmão \& Cia, Rio de Janeiro, 220 p.

Roshdy, M.A.; R. F. Foelix \& R. C. Axtell. 1972. The subgenus Persicargas (Ixodida, Argasidae, Argas). Fine structure of Haller's organ and associated tarsal setae of adults A. arboreus Kaiser, Hoogstral \& Kohls. Journal Parasitology, 58: 805-816.

Sant'Anna, M. M.; M. Amorim \& G. S. Gazêta. 2003. Análise do número de cerdas internas no Órgão de Haller de larvas de Anocentor nitens (Neumann, 1897) (Acari: Ixodidae). Entomología y Vectores. 10: $99-108$.

Serra-Freire, N. M. 2002. Planejamento e Análise de Pesquisas Parasitológicas. Rio de Janeiro: Editora Universidade Federal Fluminense, 199 p.

Serra-Freire, N. M.; B. T. M. Peixoto; V. L. Oliveira \& R. H. Teixeira. 1993. Amblyomma nodosum Neumann, 1899: contribuição ao estudo morfológico de machos e fêmeas. Revista Brasileira de Parasitologia Veterinária 2: 105-108.

Sonenshine, D. E. 1991. Biology of ticks. Vol. 1. Oxford University Press, New York, 447 p.

Vogelsang, E. G \& J. A. T. Santos Dias. 1953. Contribuccion al estudio de la fauna ixodológica de Venezuela. Revista de Medicina y Parasitología 12: 3-62.

Wooley, T. A. 1988. A Acarology-Mites and Human welfare. A Wiley-Interscience Publication, Wiley. J. \& Sons Ed., New York, 484 pp. 